\title{
Developing an evidence-based approach to Public Health Nutrition: translating evidence into policy
}

\author{
Barrie Margetts ${ }^{1, *}$, Daniel Warm ${ }^{1}$, Agneta Yngve ${ }^{2}$ and Michael Sjöström² \\ ${ }^{1}$ Public Health Nutrition, Institute of Human Nutrition, University of Southampton, Southampton SO16 6YD, UK: \\ ${ }^{2}$ Unit for Preventive Nutrition, Karolinska Institute, Stockholm, Sweden
}

\begin{abstract}
The aim of this paper is to highlight the importance of an evidence-based approach to the development, implementation and evaluation of policies aimed at improving nutrition-related health in the population. Public Health Nutrition was established to realise a population-level approach to the prevention of the major nutrition-related health problems world-wide. The scope is broad and integrates activity from local, national, regional and international levels. The aim is to inform and develop coherent and effective policies that address the key rate-limiting steps critical to improving nutrition-related public health. This paper sets out the rationale for an evidence-based approach to Public Health Nutrition developed under the umbrella of the European Network for Public Health Nutrition.
\end{abstract}

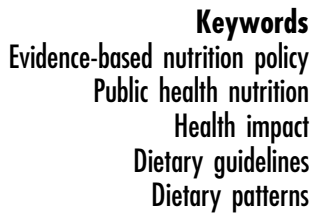

\section{Rationale}

Nutrition-related health problems such as cancer and heart disease are the major public health concerns in Europe, and increasingly beyond Europe. It is important to acknowledge that life expectancy has increased over the last 20 years in most countries in Europe $^{1}$ and throughout the world, except for areas where the effects of HIV/AIDS has led to a substantial reduction in subSaharan African countries ${ }^{2}$. Some countries in Eastern Europe have seen declines in life expectancy ${ }^{3}$. Improvements in life expectancy have not been matched by improvements in measures of the quality of life; there is a need to balance concern about quantity of life with quality of life ${ }^{2}$. There is variation in measures of health (equates to both quantity and quality) both between and within countries in Europe. In the UK, for example, there are wide regional variations in standardised mortality ratios (SMRs) and, when people are classified according to occupation or education level, there are also wide differences in measures of health between the better and worse educated. Furthermore, these socio-economic differences in health appear to have increased over time ${ }^{4}$. Dietary patterns are less likely to comply with current recommended guidelines among the poor or socially disadvantaged ${ }^{5}$.

Poor nutrition is related to the incidence of most major chronic diseases. It can be considered as a complex mix of overnutrition (obesity, inactivity), undernutrition (underweight and micronutrient deficiencies) and imbalances (type of fat, fibre, fruit and vegetable intake), such that an overweight subject may have low intakes of essential protective elements found in fruit and vegetables while still eating more energy than required. The concern then has to be about ways to ensure the best quantity and quality of the food supply to maximise individual and collective choices to eat a more 'healthy diet'.

Dietary patterns and the basis for individuals and populations to select the foods they choose to eat are complex. When developing guidelines about diet it is not possible simply to say avoid certain foods, as no foods eaten as part of a varied and balanced diet could be considered 'bad'. Unlike other lifestyle factors such as physical activity, smoking and alcohol, it is not possible to have such simple, clear guidelines (don't smoke). For physical activity the advice to do more is a clear and unambiguous message. Most current dietary guidelines are broad and non-specific and can be ambiguous. A nutrient goal, of say level of saturated fat as a percentage of energy, is a complex measure that most people could not easily work out, and therefore find hard to know whether they are meeting the guideline or not.

The factors affecting nutrition are complex and summarised in Fig. 1. If individuals do not have physical or financial access to foods recommended in dietary guidelines then it is unlikely that dietary patterns will change. The wider global and ecological issues that impact upon the food supply must also be taken into account. For example, the current push to increased olive oil consumption is not sustainable under current production capacity - the ecological consequences of expanding demand need to be considered. It is obvious that food 


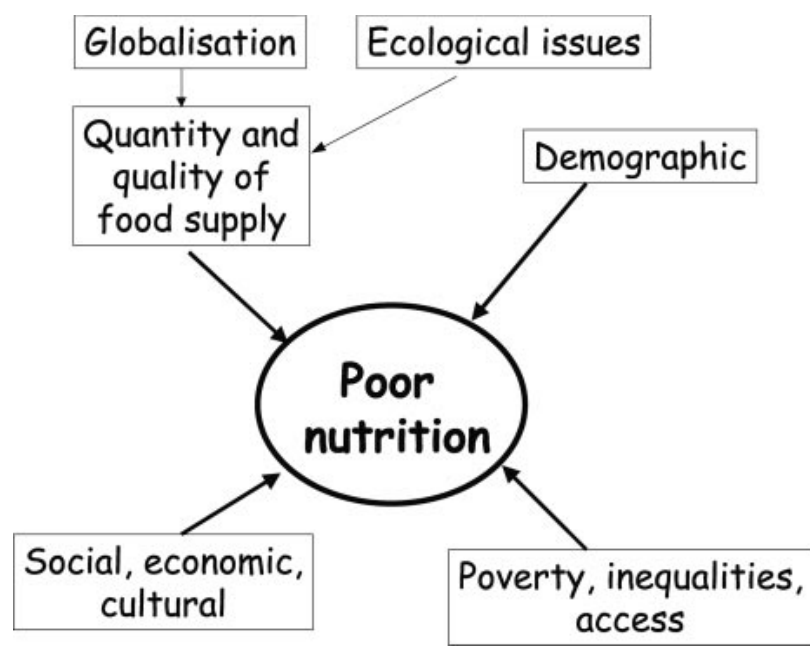

Fig. 1 Factors that influence nutrition

plays an important role beyond providing sustenance, and any programmes aimed at changing dietary patterns need to consider the social and cultural acceptability of the proposed changes. Policies aimed at improving poor nutrition need to take a wide view, and to balance the burden of responsibility between the individual and society (Fig. 2). If individuals are constrained in making healthier choices by issues of access (physical or economic), changing knowledge and attitudes will have little impact on behaviour. Ultimately, however, for population distributions of, for example, fruit and vegetables, to change for the better individuals have to change their own behaviour. For effective policy there is a need to identify and intervene at the most critical ratelimiting level and for programmes to be complementary, supportive and, most importantly, co-ordinated.

A nutritional approach needs to consider the supply of foods available, as well as the metabolic consequences of consumption of those foods - improved health can only be achieved by understanding the interaction between dietary supply and metabolic demands. Programmes that only focus on changing the food supply, without

\section{Individual Society -knowledge -responsibility -attitudes -behaviour -equity -access}

\section{Food and nutrition policy}

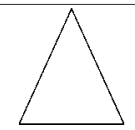

Fig. 2 Balance between individual and societal responsibilities in policy

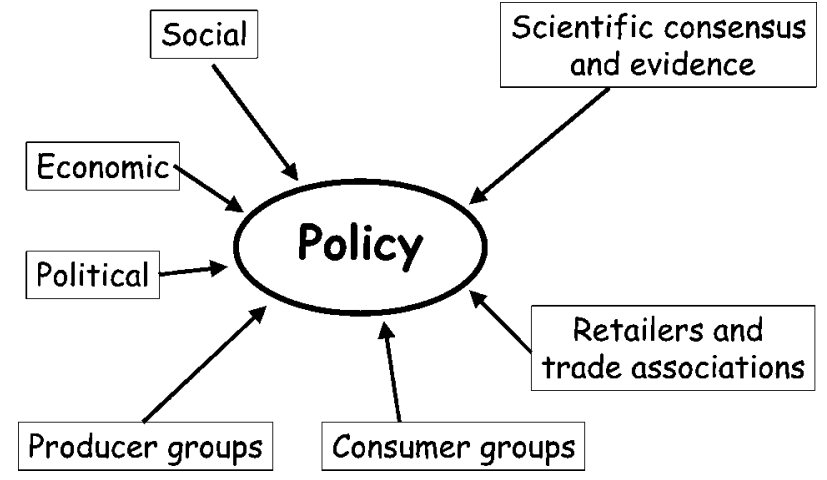

Fig. 3 Determinants of policy

considering whether people can eat the food (income, cultural acceptability, etc.), and without a consideration of the interactions of effects of different aspects of diet on metabolism and health, will be unlikely to succeed.

\section{Determinants of policy}

The main thrust of this paper is to assert that what is required is threefold - (1) an evidence-based approach to identification of the key dietary factors, (2) an objective approach to achieving scientific consensus and (3) identification of the best way to achieve the identified desirable changes. It would be naive to assume that the scientific evidence alone would determine policy.

Figure 3 summarises some of the factors that influence policy, alongside the scientific evidence. Ultimately governments are responsible for establishing and implementing policy, although there are many vested-interest groups (lobby groups) that try to influence policy. There is also a need to balance the concerns about health with safety. This is influenced by public perceptions of risk. It is clear that people are more worried about matters of safety in terms of what happens to their food and how it is produced (genetic modification, BSE, etc.) than about the consequences of the foods on their long-term health (for example, beef being safe or free from BSE and beef being 'healthy' in terms of risk of cancer). If people feel the government is not telling the truth or if someone else is interfering with their food and they feel that they have no control over food supply, they are much more likely to be concerned than if they themselves choose to undertake 'risky' behaviour (like smoking).

The public perception of risk influences the priorities governments place on different policies - policies where the government can see and show benefit in the short term (political expedience) will be more likely to be supported than policies that may take a long time to see benefits. Within the area of health the emphasis is still very much on illness and being seen to provide services in hospitals and for the care of people once they are ill.

Relatively little money and support is given to 
programmes of health promotion and prevention because the benefit of these programmes is not obvious to the public who have been prevented from having an illness. It is more difficult for politicians to get credit for long-term preventive measures that may take 20 years to affect rates of disease.

Thus, for the development of nutrition policies aimed at the promotion of health, there are two competing priorities that push resources and political will away public concerns about food safety and concerns about getting treated when they become ill. Vested interests exploit this situation to their own ends, and generally at the expense of taking the long-term public health view of what is best for the majority of the population.

Part of the lack of resolve of governments to develop public health nutrition policies may also arise from an apparent lack of agreement about what the policy should contain. If governments seek guidance on what they should do to improve nutrition, and there is no broad agreement as to the best things to do, why should governments develop a policy? A lack of consensus on the evidence and most effective actions does not help, and hence the need for an evidence-based approach. This makes it even more important that when competing interests seek to alter policy and use lack of evidence or quality of evidence as a reason that this is itself based on evidence. In other words, it is important that there is little or no room for debate about what the evidence is saying, given the pressure on governments not to be involved in health promotion anyway.

The position as to whether to have a policy, and the nature and content of that policy, is a function of a complex interaction, as shown in Fig. 3. This also includes an interaction between the facts (evidence), the beliefs and values of the people involved in the process, and the goals of the policy.

\section{Policy cycle}

Figure 4 presents a schematic model of the policy cycle. This shows that the policy cycle should be driven by an evidence-based approach to identify the key preventable nutrition-related health problems that should be addressed and would be likely to lead to health improvement in the population. There is no point identifying problems for which there is no solution, or where eliminating the problem would have no impact on public health. Goals for action must emerge out of this review and guide subsequent objectives and programmes of work. There will be options for actions that need to be weighed up for cost benefit/effectiveness and for potential adverse effects. A clear programme of implementation is then required that considers all of the practical details required to deliver the programme of work. The programme should then be evaluated and the results fed back to inform the decision-making process

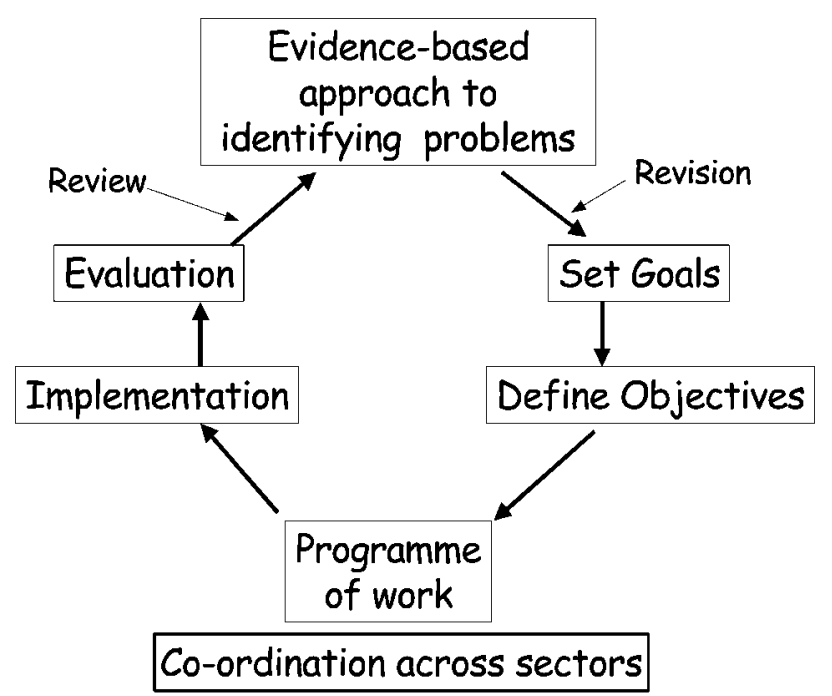

Fig. 4 The policy cycle

and to subsequently revise goals. Evaluation should consider both process (delivery) and outcome (behaviour).

The other aspect of the policy cycle is the need for considering how best to co-ordinate activity across different departments of government, voluntary agencies and other groups. Ideally all groups with a vested interest need to work together in a co-ordinated, integrated fashion.

\section{Evidence-based policy}

Whilst there is broad general agreement about the type of diet that is related to reduced risk of disease, when it comes down to the detail there is less consensus. Part of the reason for this is that, until recently, there was little agreement about the optimal approach to reviewing and synthesising the evidence in a way that leads to consensus on what the evidence suggests as a basis for policy. Without agreement about what the evidence says, it is difficult to get conformity about the correct approach to improving the nutritionrelated health of the population. Most countries have dietary recommendations that are broadly similar and aimed at reducing the burden of chronic diseases. There is less clarity as to how these recommendations are used and implemented to lead to the desired changes. There is a need for agreement and evidence, to support the best approaches to achieving desired dietary changes. It is therefore critical to ensure that:

- interventions or actions are based on the best possible evidence;

- approaches to achieve objectives that are more likely to work are used; and

- there are defined, measurable and clear objectives, which will aid in making it easier to evaluate impact.

\section{The derivation of evidence-based policy}

In order for the derivation of evidence-based policy, it is 
critical to ensure that the policy is based on three types of evidence, namely:

1. evidence about the scientific basis of recommendations;

2. evidence about strategies that work (past experience); and

3. evidence of policy impact (does it lead to desirable changes?).

Evidence about the scientific basis of recommendations There is now wide agreement about the ideal approach to gathering and reviewing evidence. Considerable international effort has been devoted to drawing together the evidence from randomised controlled trials for the effectiveness of a whole range of interventions. This model of review and synthesis is ideal for single nutrient or drug-type secondary prevention trials, but is more problematic for dietary studies and primary prevention trials where it is difficult to control all aspects of dietary change and to conduct a double blind placebo controlled trial. From a public health point of view, we are interested in understanding the links between what people eat and their health.

Understanding the effects of nutrients or compounds within foods (phytochemicals) studied in isolation is not necessarily helpful in understanding the interaction between nutrients and the effect changes in diet may have on health. People who consume more fruit and vegetables also tend to eat less animal products; attributing the protective effect of this dietary pattern to vegetable intake - or, even further, vitamin $\mathrm{C}$ intake is likely to be simplistic and misleading. Therefore, the approach to be taken in reviewing the scientific evidence needs to take into account the complexity of diet as the exposure. The quality of the assessment of diet needs to be taken into account when judging the scientific quality of evidence. Nevertheless, having said the above, there are guidelines for ways to gather epidemiological literature and review the evidence from observational research.

These guidelines may be summarised as follows.

- Judgement based on systematic review of all available literature.

- Explicit search strategy and inclusion criteria.

- Review guided by:

- type of epidemiological study;

- consistency of results between studies;

- quality of studies reviewed;

- validity of measure of exposure;

- consideration of the effects of

- sampling, sample size and power

- chance, bias and confounding;

- study-design-specific issues (referral group, loss to follow-up, etc.);
- size of risk estimate;

- evidence that the exposure precedes the outcome; and

- plausible mechanisms.

If the above approach were used in a logical and consistent manner for all reviews of the relationship between diet and health, it would be a great deal easier to assess the strength of the evidence to support policy. Drawing causal inferences from epidemiological studies is fraught with the possibility of error, whereas wellconducted studies, clearly presented and not overinterpreted can provide helpful evidence to guide judgement as to the likely role of diet in affecting health. Where disease end points are not studied, but risk factors (e.g. lipids, blood pressure, obesity) are, it is important to be clear about the limits of the data. When making policy from inevitably imperfect data, it is also important to consider the axiom of doing no harm whilst trying to do good. Not drawing conclusions from the evidence may have effects on health that are worse than drawing conclusions that may only be partly correct.

\section{Evidence about strategies that work}

Having reached some agreement on the desired goals of the policy based on the review of the scientific evidence, evidence is also required on strategies that work to achieve (or in some cases maintain) the desired dietary patterns. It is critical that strategies are based on evidence otherwise they are potentially open to the same difficulties and criticisms as non-evidence-based policies. Therefore, adherence to the following criteria is needed in order to consider the evidence regarding strategies:

- the approach should be based on principles of Health Promotion;

- consider an approach that is more than providing information;

- consider the wider social, economic and cultural context;

- allow for the measurement of process and outcome indicators;

- consider the need for different approaches in different groups and settings; and

- consider both cost-effectiveness and cost benefit.

In judging whether a policy works it is important to have success criteria (indicators) that can be measured (with the required level of accuracy) and can be clearly linked to the explicit goals of the policy. Indicators and goals/targets should be:

- credible (address important issues);

- clear (easily appraised);

- selective (highlight priority);

- compatible with other and current strategies;

- achievable;

- balanced; 


\section{Draft position statement on food and nutrition policies}

- Be driven by health

- Be designed to reduce nutrition-related health inequalities

- Be based on evidence of what works

- Be evaluated for impact

* Be supported by well-trained staff

- Consider ecological sustainability

* Consider cultural diversity

Fig. 5 Draft statement on food and nutrition policies proposed by The European Network for Public Health Nutrition

- quantifiable; and

- ethical.

\section{Evidence of policy impact}

The third level of evidence that is required to drive an effective policy cycle is to assess whether the policy itself is leading to the desired changes outlined in the same said policy. Changes may appear to be due to the policy, but may in fact be due to other factors that have changed. It is therefore necessary to assess what effect the policy itself is having. There is a need for evidence that a policy is having an effect.

- Is the policy clear and does it identify measurable goals?

- Does it achieve its objectives?

- What is the impact on health?

- Is it cost-effective?
The European Network for Public Healtb Nutrition This network was established to co-ordinate Public Health Nutrition, education, training and research across Europe $^{6,7}$. It has recently proposed a draft statement on food and nutrition policies (Fig. 5). This statement emphasises that the policy should be driven by health and be designed to reduce health inequalities, and that any policy should be based on evidence. The aim of this draft is to draw emphasis away from policies that focus on food and agricultural production in isolation from a consideration of the impact on health. Food policy that focuses on matters of production, resources used in production, and marketing and consumption may not focus on the key rate-limiting steps in improving nutrition-related health.

\section{References}

1 European Commission. The State of Health in the European Community. 1996. Luxembourg: Office for Publications of the European Community, 1997.

2 World Health Organization (WHO). The World Health Report 1997: Conquering Suffering Enriching Humanity. Geneva: WHO, 1997.

3 Ferro-Luzzi A, James WPT. Diet and health: brief summary of our present knowledge and description of the present European situation in terms of diet, health and their relationship. In: Trichopoulou A, ed. Nutrition in Europe. Luxembourg: European Parliament, 1997; 2-38.

4 Acheson D, Barker D, Chambers J, Graham H, Marmot M, Whitehead M. Independent Inquiry into Inequalities in Health Report. London: The Stationery Office, 1998.

5 Department of Health. Low Income, Food, Nutrition and Health: Strategies for Improvement. London: HMSO, 1996.

6 Sjostrom M, Yngve A, Poortvliet E, Warm D, Ekelund U. Diet and physical activity - interactions for health; public health nutrition in the European perspective. Public Health Nutr. 1999; 2(3A): 453-9.

7 Yngve A, Sjostrom M, Warm D, Margetts B, Rodrigo CP, Nissinen A. Effective promotion of healthy nutrition and physical activity in Europe requires skilled and competent people; European Master's Programme in Public Health Nutrition. Public Health Nutr. 1999; 2(3A): 449-52. 